\title{
Los derechos culturales: Una categoría aún subestimada de derechos humanos
}

\author{
Cultural rights: A still over-looked category of human rights
}

Andrés Muñoz Cárcamo

Universidad de Chile

\begin{abstract}
RESUMEN Este artículo busca presentar una lectura en torno a los derechos culturales, derechos humanos que siguen reclamando mayor atención. Se apunta a su complejidad conceptual, derivada de la amplitud de significados de la cultura. Se propone aproximarse a ellos mediante la idea de «derechos expresamente culturales». Bajo este conjunto acotado de derechos humanos es posible agrupar una vasta extensión de asuntos culturales de relevancia jurídica. Se abordan someramente las nociones de identidades, diversidad y patrimonio cultural, que figuran como partes claves del lenguaje de los derechos culturales. Adicionalmente, se vincula la dimensión colectiva de la cultura con el ejercicio en grupo o comunidad de los derechos individuales, así como se alude a la espinosa cuestión de los derechos colectivos, todo lo cual es relevante para los pueblos indígenas y las minorías culturales. Finalmente, se trata con mayor detención el derecho a participar en la vida cultural, aquel cuyo contenido se aprecia como el más extensivo de este catálogo, y se describen aspectos de su alcance, en particular las obligaciones dirigidas a los Estados en orden a su respeto, protección y satisfacción. Este análisis puede ofrecer luces para la interpretación de la categoría completa de derechos culturales.
\end{abstract}

PALABRAS CLAVE Derechos culturales, derecho a participar en la vida cultural, derechos humanos, derecho internacional de los derechos humanos.

ABSTRACT The present article aims to provide an interpretative view of cultural rights, human rights that still demand greater attention. These rights are conceptually challenging, since the idea of culture has a vast scope and is hard to define. Nonetheless, it is proposed to conceive them as "express cultural rights". Within these specific rights there is a wide range of legally relevant cultural issues that interrelate. The notions of identities, diversity and cultural heritage, key parts of the broad sense of culture, are briefly addressed. In addition, the collective dimension of culture is associated with the group exercise of individual rights. The thorny issue of collective rights is also brought up. This is relevant to indigenous peoples and cultural minorities. Finally, certain aspects of the right to take part in cultural life, which is seen as the most comprehensive of cultural 
rights, are dealt in greater depth. In particular, emphasis is given to the obligations that concern the States to respect, protect and fulfill this right. This analysis might provide insights for the scope of the entire category of cultural rights.

KEYWORDS Cultural rights, right to participate in cultural life, human rights, international human rights law.

\section{Introducción: Dificultades conceptuales}

No es una tarea sencilla precisar en qué consisten los derechos culturales. No son nuevos derechos: el derecho a participar en la vida cultural y los demás derechos en la esfera de la cultura han sido recogidos desde la Declaración Americana de Derechos y Deberes del Hombre de 1948. La Declaración Universal de Derechos Humanos, adoptada solo meses después, establece en su artículo 27 esta categoría, partiendo por proclamar el «derecho de tomar parte libremente en la vida cultural». ${ }^{1}$ Ambas declaraciones cumplen ya 70 años de historia.

Aun cuando integran la familia de derechos humanos desde entonces, los derechos culturales no han suscitado el mismo grado de desarrollo, promoción e implementación que otros derechos esenciales. Tanto es así, que la primera investigación académica seriamente dedicada a ellos, publicada hace 25 años, se tituló «Derechos culturales: Una categoría subdesarrollada de derechos humanos». ${ }^{2}$ Esta calificación acertó en señalar la falta de elucidación respecto de su alcance, contenido normativo y cómo hacerlos efectivos, razón por la cual han sido vistos como «parientes pobres» de los derechos humanos (Symonides, 1998: 559). Esta situación de descuido, que en menor medida aún se advierte, obedece a distintas razones. En parte, porque se trata de derechos vinculados a la cultura. Ello supone lidiar con una idea tan vasta como compleja.

«Cultura» puede referir a muchas cosas, desde el cultivo de las ciencias y disciplinas del conocimiento, incluidas las artes y letras, hasta un proceso integral asociado

\footnotetext{
1. A diferencia de la redacción en español, el artículo 27 de la Declaración Universal en lengua inglesa se refiere al derecho «to participate in cultural life». Curiosamente, una disparidad equivalente se da en el Pacto Internacional de Derechos Económicos, Sociales y Culturales, solo que en sentido contrario, pues mientras el texto del Pacto en español expresa que los Estados reconocen el derecho de toda persona a «participar en la vida cultural», en inglés dice «to take part in cultural life».

2. Corresponde a la compilación de las actas y documentos resultantes de la octava versión del coloquio del Centro Interdisciplinario de Ética y Derechos Humanos de la Universidad de Friburgo, Suiza, que estudió exclusivamente estos derechos. Se publicó en 1993 bajo la edición de Patrice Meyer-Bisch y su título original en francés es Les droits culturels: Une catégorie sous-développée de droits de l'homme. A partir de este hito, alrededor del mencionado centro académico, se fraguó el grupo de trabajo de investigadores que redactó la Declaración de Friburgo sobre los Derechos Culturales de 2007.
} 
a las formas de vidas, pasando por las entidades establecidas para su transferencia, como los museos, las instituciones educativas y los medios de comunicación. Esta indeterminación ha llevado a una falta de consenso sobre qué derechos son culturales y cómo deben implementarse (Donders, 2008). De cualquier manera, se hace necesario considerar un significado general de cultura.

Una difundida definición ha propuesto entenderla como «conjunto de los rasgos distintivos, espirituales y materiales, intelectuales y afectivos que caracterizan a una sociedad o un grupo social», agregando que abarca «además de las artes y las letras, los modos de vida, los derechos fundamentales al ser humano, los sistemas de valores, las tradiciones y las creencias». ${ }^{3}$ Este concepto abierto remite a la antropología, a las maneras de vivir y formas de expresión de los seres humanos, tanto en cuanto individuos como en una dimensión colectiva. Este carácter holístico importa complejidades lógicas para la circunscripción de los derechos culturales. Sin embargo, el adecuado entendimiento de esta categoría debiese partir sobre una mirada amplia de la cultura.

Con todo, es posible apreciar en los últimos años un renovado interés en los derechos culturales. Se cuentan algunas iniciativas significativas, provenientes de sectores de la sociedad civil y la academia, así como de la comunidad internacional organizada. Entre ellas está la Carta Cultural Iberoamericana, declaración aprobada por la Comunidad Iberoamericana de Naciones en 2006, que rescata los derechos culturales como derechos de carácter fundamental. ${ }^{4}$ Otro hito en esta dirección es la Declaración de Friburgo sobre los Derechos Culturales de 2007, documento no vinculante que propone un catálogo ampliado de derechos culturales y que constituye un notable esfuerzo en aras de esclarecer y divulgar estos derechos. ${ }^{5}$

3. Unesco, «Declaración de México sobre las Políticas Culturales», aprobada tras la Conferencia Mundial de las Políticas Culturales, 1982, página 1, disponible en http://bit.ly/2CGwDxI.

4. Este instrumento fue adoptado tras la Decimosexta Cumbre Iberoamericana celebrada en Montevideo y es fruto de un esfuerzo conducido por la Organización de Estados Iberoamericanos para la Educación, la Ciencia y la Cultura (OEI). Estructurada en un preámbulo y en cuatro títulos relativos a los fines, principios, ámbitos de aplicación y al «espacio cultural iberoamericano», esta declaración asume un significado abierto de cultura como «base indispensable para el desarrollo integral del ser humano y para la superación de la pobreza y de la desigualdad» y reafirma un principio de «reconocimiento y protección de los derechos culturales». («Carta Cultural Iberoamericana», Organización de Estados Iberoamericanos, 2006, disponible en http://bit.ly/2ReoAMD).

5. En su preámbulo, la Declaración de Friburgo reafirma que los derechos culturales son «expresión y exigencia de la dignidad humana». Luego, enuncia principios fundamentales —en primer lugar, la no discriminación en el ejercicio de los derechos culturales-y define algunos términos relevantes, expresando que la palabra cultura comprende «los valores, las creencias, las convicciones, los idiomas, los saberes y las artes, las tradiciones, instituciones y modos de vida por medio de los cuales una persona o un grupo expresa su humanidad y los significados que da a su existencia y a su desarrollo». Sobre la base de esta definición, y a lo largo de sus doce artículos, la declaración reúne y busca explicitar derechos 
$\mathrm{Al}$ margen de la permanente - y a veces subestimada - labor de la Unesco en estos asuntos, la Asamblea General de las Naciones Unidas aprobó en 2009 la Observación General 21 del Comité de Derechos Económicos, Sociales y Culturales, referida al derecho de toda persona a participar en la vida cultural. Además, ese mismo año se fijó el mandato de «experta independiente en la esfera de los derechos culturales», renovado en 2012 y renombrado como «relatora especial». Estas dos medidas representan importantes avances desde los órganos internacionales de derechos humanos. El trabajo de la relatora, a través de sus informes periódicos, ha contribuido fuertemente a clarificar la envergadura de los derechos culturales y a visibilizar su relevancia.

Los derechos culturales empiezan al fin a revertir su descuidada situación. Hay mayor noción de su existencia y de su calidad de derechos universales. Sin embargo, aún queda mucho por hacer para implementarlos efectivamente. La actual relatora especial, Farida Shaheed, ha afirmado que si bien han adquirido legitimidad, persiste «la creencia generalizada de que los derechos culturales son un lujo». ${ }^{6}$ Esto alude indirectamente a un entendimiento según el cual estos derechos son costosos y dependientes de la capacidad económica de los Estados. Entonces, parece paradójico que, vistos como el pariente pobre de los derechos humanos, los derechos culturales sean además pensados como derechos caros.

Existe todavía una visión que distingue fuertemente entre derechos fundamentales según la actuación que le correspondería al Estado en cada caso. De una parte, estarían los derechos civiles y políticos, que exigen abstención estatal y, por otra, los económicos, sociales y culturales, que requieren de capacidad del Estado. ${ }^{7}$ Esta

reconocidos «de manera dispersa en un gran número de instrumentos de derechos humanos», con el propósito de demostrar su importancia, como también la de «las dimensiones culturales de los demás derechos humanos». Documento disponible en http://bit.ly/2ReB32M.

6. «Informe periódico de la relatora especial en la esfera de los derechos culturales», Naciones Unidas, $\mathrm{A} / \mathrm{HRC} / 31 / 59,3$ de febrero de 2016, párrafo 5, disponible en http://bit.ly/2RhV9JA.

7. Esta lectura proviene de la largamente difundida tesis de las tres generaciones de derechos humanos (Vašák, 1977). Según la formulación original de esta propuesta, la primera generación de derechos, los civiles y políticos, han de ser vistos como derechos «atributos», en el sentido de ser oponibles al Estado, al que corresponde una obligación negativa de no interferencia. Por el contrario, la segunda generación, los económicos, sociales y culturales, son derechos «de crédito», que requieren del Estado acciones positivas y progresivas para su realización. Por último, la tercera generación se refiere a los «derechos de solidaridad», entre los que se encontrarían el derecho al desarrollo y el derecho a un medioambiente sano. Aunque esta tesis ha contribuido a educar sobre derechos humanos mediante una presentación pedagógica y sencilla, ha pavimentado el erróneo entendimiento conforme al cual solo los civiles y políticos serían derechos en un sentido estricto, mientras que los derechos económicos, sociales y culturales serían derechos de segunda clase, en el mejor caso solo programáticos. Esto ciertamente pugna con los principios de universalidad, indivisibilidad, interdependencia e interrelación del conjunto de derechos humanos y elude la variedad de obligaciones que cada derecho humano conlleva para los Estados. 
inexacta interpretación debiese ser superada por una comprensión más cabal de la familia de derechos humanos como derechos que requieren tanto de medidas negativas como positivas para su completa realización.

\section{Derechos expresamente culturales}

¿Qué derechos culturales? Como se ha anticipado, entre la reducida doctrina que los ha estudiado no hay acuerdo sobre cuántos derechos son. Por ejemplo, Symonides (1993: 52-62) sostuvo que esta categoría de derechos humanos se compone de aquellos mencionados en el artículo 27 de la Declaración Universal, sumado a los derechos a la educación y a la información. Pero advierte que si se consideraran los instrumentos de la Unesco, se puede pensar en un catálogo abierto de derechos culturales que incorpora los derechos de acceso a la cultura; a la creatividad; a la protección y desarrollo de la identidad cultural; a la protección de los intereses morales y materiales que resultan de la producción literaria o artística; a la protección de los bienes culturales y del patrimonio cultural mundial y, además, el derecho de los Estados a la cooperación cultural internacional.

Por su parte, Prieto de Pedro (2008: 20-21) señala que una «concepción integral de los derechos culturales» debiese contemplar la totalidad de los derechos que se relacionan con los procesos culturales, comprendiendo así a las libertades de creación artística, científica y de comunicación cultural; los derechos de autor; el derecho de acceso a la cultura; el derecho a la identidad y a la diferencia cultural; y el derecho a la conservación de la cultura.

Mientras, Stamatopoulou-Robbins (2008: 3) ha afirmado que en el derecho internacional de los derechos humanos son generalmente cinco los entendidos como derechos culturales: el derecho de educación; el derecho a participar en la vida cultural; el derecho de disfrutar de los beneficios del progreso científico y sus aplicaciones; el derecho de beneficiarse de la protección de los intereses morales y materiales que le correspondan por razón de las producciones científicas, literarias o artísticas de que sea autor y, finalmente, la libertad para la investigación científica y la actividad creativa.

Dada la omnipresencia de la dimensión de la cultura en todas las esferas de la vida en comunidad, ella se manifiesta en el ejercicio de todos los derechos humanos. Los derechos culturales presentan así un «carácter transversal», en el sentido de que pueden «solaparse» en las categorías de derechos económicos, sociales, civiles y políticos (Donders, 2004: 155). Esta característica de lo cultural complejiza los límites de la categoría de derechos correspondiente, por lo que determinar cuántos forman parte de ésta resulta un asunto abierto al debate y a la interpretación jurídica.

Además, omite que todo derecho humano exige un nivel mínimo de satisfacción, esto es, un contenido básico que no puede estar condicionado por razones económicas. 
Se propone aquí concebir los derechos culturales en términos de las referencias explícitas a la esfera de la cultura presentes en la Carta Internacional de Derechos Humanos. Se asumen por referencias explícitas a la cultura las disposiciones contenidas en el artículo 27 de la Declaración Universal de los Derechos Humanos y el artículo 15 del Pacto Internacional de Derechos Económicos, Sociales y Culturales. Además de estas normas, las referencias de los artículos inmediatamente precedentes en ambos instrumentos que recogen el derecho a la educación deben entenderse añadidas (artículo 26 de la Declaración y los artículos 13 y 14 del Pacto).

De la lectura de las normas señaladas en el párrafo anterior se desprende el siguiente catálogo acotado de «derechos expresamente culturales»: el derecho a participar en la vida cultural (enunciación que puede abreviarse como «derecho a la cultura»); el derecho de toda persona a participar en el progreso científico y sus beneficios («derecho a la ciencia»); el derecho de toda persona a beneficiarse de la protección de los intereses morales y materiales que le correspondan por razón de las producciones científicas, literarias o artísticas de que sea autor («derecho a la protección de la autoría») y, además, el «derecho a la educación». ${ }^{8}$

El derecho a la educación se agrega así a los derechos expresados en el artículo 15 del Pacto Internacional de Derechos Económicos, Sociales y Culturales, norma que establece el «marco general y normativo para un catálogo de los derechos culturales» (Nieć, 2001: 290). La calidad cultural del derecho a la educación suele ser pasada por alto. Se lo concibe más bien como derecho social, pero la precedencia del derecho a la educación a las referencias explícitas de la cultura dentro del texto normativo no es mera casualidad. Hay una estrecha relación entre estas estructuras de la vida en comunidad; aún más, la educación puede ser vista como el medio de la cultura. Hay entonces una razón de fondo para comprender el derecho a la educación como derecho cultural. ${ }^{9}$

Esta interpretación se encuentra respaldada por la Declaración Universal sobre la Diversidad Cultural, adoptada por la Conferencia Mundial de la Unesco en 2001.

8. Además de encontrarse presentes en los artículos ya mencionados de la Carta Internacional de los Derechos Humanos, estos derechos son reconocidos en instrumentos regionales de derechos humanos, como la Declaración Americana de Derechos y Deberes del Hombre (artículos 12 y 13) y la Carta Africana sobre Derechos Humanos y de los Pueblos (fundamentalmente el artículo 17). Además, una diversidad de convenciones internacionales de derechos humanos alude a ellos. Para una referencia detallada de las disposiciones normativas del derecho internacional relativas a cada uno de estos derechos culturales, véase Muñoz Cárcamo (2016: 64-78).

9. La educación puede entenderse como el canal a través del cual se transmiten conocimientos, creencias, costumbres, lenguas y otros elementos culturales que permiten a las personas comprender la cultura propia y aproximarse a la cultura de otros. Es a través de la educación que el ser humano tiene la posibilidad de acceder a los valores y bienes de la cultura, así como a desarrollar sus propias capacidades y visiones de mundo. 
En su artículo 5, referido a los derechos culturales como «marco propicio para la diversidad cultural», expresa que el desarrollo de una «diversidad creativa» exige la «plena realización de los derechos culturales, como los definen el artículo 27 de la Declaración Universal de los Derechos Humanos y los artículos 13 y 15 del Pacto Internacional de Derechos Económicos, Sociales y Culturales»..$^{10}$ Cabe agregar que a excepción del derecho a la ciencia, estos derechos - en términos de cómo están dispuestos en el Pacto respectivo- han sido objeto de análisis interpretativo por parte del Comité de Derechos Económicos, Sociales y Culturales a través de sus Observaciones Generales. ${ }^{11}$

Al tratarse de un catálogo restringido, hay ciertas disposiciones que no se conciben en términos de derechos expresos. Es el caso de la referencia a gozar las artes, incluida en el artículo 27 de la Declaración Universal, pero omitida en el artículo 15 del Pacto Internacional. ${ }^{12}$ Esta última disposición, no obstante, expresa en su numeral 3 que los Estados se comprometen a respetar «la indispensable libertad para la investigación científica y para la actividad creadora». Esta libertad, del mismo modo que la facultad de gozar de las artes, puede comprenderse como un elemento subsumido dentro del conjunto de derechos propuesto. En efecto, la posibilidad de crear arte y la de gozarlo sí forman parte del derecho a tomar parte libremente en la vida cultural.

Este entendimiento permite vincular determinadas referencias implícitas a la esfera de la cultura en otras disposiciones normativas del derecho internacional de los derechos humanos. Hay normas que, sin configurar precisamente un derecho cultural, se asocian fuertemente a ellos. Por ejemplo, el «derecho a la información», desprendido de la libertad de expresión que contiene las de buscar, difundir y recibir informaciones - en los términos en que está recogido en el artículo 19 de la

10. Esta disposición luego ahonda en el contenido de esta categoría de derechos al agregar que «toda persona debe tener la posibilidad de expresarse, crear y difundir sus obras en la lengua que desee y en particular en su lengua materna; toda persona tiene derecho a una educación y una formación de calidad que respeten plenamente su identidad cultural; toda persona debe tener la posibilidad de participar en la vida cultural que elija y conformarse a las prácticas de su propia cultura, dentro de los límites que impone el respeto de los derechos humanos y de las libertades fundamentales».

11. Se trata de la Observación General 13 de 1999 sobre el derecho a la educación; Observación General 17 de 2006 sobre el derecho a beneficiarse de la protección de los intereses morales y materiales que le correspondan por razón de las producciones científicas, literarias o artísticas de que sea autor, y Observación General 21 de 2009, sobre el derecho a participar en la vida cultural. Se espera que la observación general sobre el derecho a gozar de los beneficios del progreso científico y de sus aplicaciones salga a la luz en una próxima sesión del Comité.

12. Hay razones adicionales a la falta de inclusión en el texto del Pacto que cuestionan la proyección de un «derecho humano al arte». Pensarlo como un derecho implicaría asegurar la existencia del arte. Determinar qué es el arte o cuándo estamos ante una obra de arte puede resultar muy arbitrario o subjetivo. Teixeira Coelho (2008: 132) ha señalado que establecer tal derecho «es algo muy complejo, puede pensarse como un privilegio y no un derecho». 
Declaración Universal y en el artículo de mismo número del Pacto Internacional de Derechos Civiles y Políticos-, en ocasiones ha sido incluido como derecho cultural. Sin embargo, bajo la interpretación que aquí se sugiere debiese encuadrarse más bien como un derecho civil, pero con la prevención de que, atendiendo a los principios de interrelación e interdependencia de los derechos humanos, el ámbito de acción de dicha libertad no se circunscribe exclusivamente a la esfera civil. En realidad, los derechos culturales no pueden ser ejercitados sin el acceso a la información.

A partir de estos cuatro derechos expresamente culturales es posible examinar una amplia variedad de asuntos en la esfera de la cultura que reportan relevancia jurídica. Esta categoría de derechos humanos permite guiar la acción de los Estados y sus ordenamientos jurídicos en relación con el respeto por los diversos modos de vida y la formulación de visiones de mundo por parte de todas las personas; la libertad de las personas para actuar, expresarse y crear en función de sus legítimas identidades personales y grupales; la protección de la autoría sobre las obras del intelecto y el fomento a la creación e inventiva humana; la salvaguardia del acervo de bienes que forman el patrimonio cultural, material e inmaterial; el acceso a la educación, a los beneficios del progreso científico y a los bienes de la cultura; en definitiva, el acceso, contribución y participación en la vida cultural.

Del anterior espectro de cuestiones, las nociones de identidades, patrimonio y diversidad cultural merecen ser abordadas especialmente, en razón a que forman parte esencial del campo semántico de los derechos culturales. En efecto, estos términos aparecen de forma habitual en documentos y discusiones sobre esta categoría de derechos humanos y, sin embargo, se advierte que rara vez se les precisa conceptualmente. A continuación, se intenta describir estas ideas.

\section{Identidades, patrimonio y diversidad cultural}

Toda persona humana es portadora de una identidad que constituye la referencia de su individualidad. Ella se construye desde la experiencia de la realidad en la que cada sujeto se encuentra inserto, que comprende el tiempo y el lugar que lo rodea, así como el contexto social y cultural próximo. Las identidades cumplen un rol de reconocimiento dentro de las sociedades, pues permiten a los sujetos verse como seres iguales, en términos de la naturaleza y dignidad humana que comparten, y a la vez como individualidades diversas que no obstante pueden confluir en un área común de pertenencia. Así se van formando las identidades colectivas, que pueden ser entendidas como la representación de ciertos rasgos distintivos de un grupo o comunidad humana, que resultan del proceso vital de la cultura, y que son capaces de adquirir desarrollo propio e histórico en cuanto entidades colectivas. Así, mientras las identidades individuales realzan los rasgos que distinguen a una persona de otra, las identidades colectivas resaltan ciertas similitudes entre los diferentes miembros de un grupo. 
El respeto a las identidades culturales, individuales y colectivas constituye sin dudas un asunto que concierne a los derechos humanos y que atañe directamente a los derechos culturales, especialmente al derecho de participar en la vida cultural, toda vez que éste, como se verá, refiere a una libertad que se ejerce a partir de la identidad cultural. A mayor abundamiento, uno de los más prominentes miembros del Grupo de Friburgo ha formulado una definición de los derechos culturales alrededor de la idea de identidades, en términos de «los derechos de una persona, sola o en comunidad, de escoger y expresar su identidad, acceder a referencias culturales, como también a los recursos que son necesarios a su proceso de identificación» (Meyer-Bisch, 2010: 7).

El patrimonio cultural constituye asimismo parte relevante del «universo del discurso» de los derechos culturales. Este concepto guarda relación con un conjunto de bienes suficientemente trascendentes en términos de simbolizar la vida y la herencia cultural de un grupo, comunidad o pueblo. Pueden incluso llegar a constituir expresiones patrimoniales representativas de la humanidad, conforme a la idea de bienes materiales e inmateriales excepcionales según las convenciones de la Unesco. ${ }^{13}$

Los bienes que componen un patrimonio cultural hacen referencia a la cultura en el aspecto colectivo, pues tienen especial significancia para los modos de vida y rasgos distintivos de un conjunto humano. Estos bienes son considerados patrimoniales no por su utilidad económica, sino por su valor histórico, estético, arquitectónico, urbano, arqueológico o de otro orden cultural pertinente - lo que incluye el valor tradicional y simbólico de ciertos bienes intangibles-, que representa un sentido de pertenencia al grupo humano respectivo. Los bienes integrantes de un determinado patrimonio cultural son relevantes desde que pueden reflejar elementos o características distintivas de las identidades culturales, tanto individuales como colectivas. Es por ello que los Estados están llamados a proteger el patrimonio cultural que se alberga dentro de sus territorios y comunidades, así como a promover su acceso a todas las personas.

La diversidad cultural, por último, ha sido vista en términos de un valor representativo de la dignidad humana. La Declaración sobre la Diversidad Cultural de la Unesco de 2001 reconoce en su artículo 4 que los derechos humanos son garantes de la diversidad cultural y que la defensa de la diversidad constituye un «imperativo ético, inseparable del respeto de la dignidad de la persona humana», que entraña el compromiso de respetar los derechos humanos y, en particular, «los derechos de las personas que pertenecen a minorías y los de los pueblos indígenas».

Ahora bien, la diversidad cultural no es equivalente a relativismo cultural. Hay ciertas prácticas culturales que no pueden ser aceptadas ética y jurídicamente, por

13. En particular, a través de las listas representativas del patrimonio de la humanidad, material e inmaterial, en virtud de la Convención sobre la Protección del Patrimonio Mundial Cultural y Natural de 1972 y la Convención para la Salvaguardia del Patrimonio Cultural Inmaterial de 2003. 
cuanto pueden reñirse con el ejercicio mismo de los derechos humanos por parte de otras personas, y nadie puede invocar la diversidad cultural para violar estos derechos. Hay un desafío al interior de cada grupo humano - y a nivel moral, al interior de la conciencia de cada persona- de reconocer y erradicar las prácticas culturales negativas. En consecuencia, la diversidad cultural como manifestación del valor de la dignidad humana de todas las personas se debe encuadrar dentro de los márgenes de los derechos humanos, por lo que bien podría hablarse de pluralismo cultural.

La idea de pluralismo cultural, esto es, el respeto por la legítima diversidad de identidades y expresiones en la esfera de la cultura alude y abarca especialmente a las minorías culturales. Debido a la situación de discriminación y vulneraciones que históricamente han sufrido los pueblos indígenas y otras comunidades minoritarias, se justifica que los Estados pongan particular atención sobre sus derechos. Esto abre la puerta para hablar del ejercicio colectivo de los derechos humanos y culturales.

\section{La dimensión colectiva de los derechos y los derechos colectivos}

El reconocimiento de los derechos colectivos es un tema que suscita suficiente controversia jurídica, aun en la interpretación de las normas del derecho internacional. Pero se ha de distinguir la dimensión colectiva de los derechos humanos de los derechos colectivos en sí mismos, pues se han entendido como cuestiones sutilmente distintas. Mientras la dimensión colectiva de los derechos es entendida como el ejercicio colectivo de los derechos humanos individuales, los derechos colectivos son comprendidos como derechos de grupo o pertenecientes a un grupo humano determinado. ${ }^{14}$

Los derechos humanos proclamados en los instrumentos del derecho internacional de los derechos humanos se reconocen «a toda persona». Habitualmente se ha entendido esta enunciación en términos de atribuir el derecho a cada persona individualmente considerada. En ocasiones, no obstante, se ha interpretado que estos derechos son ejercitables por sujetos colectivos. En cuanto a los derechos culturales, esto se ha sostenido en relación con el derecho a la protección de la autoría, el que puede «en ciertas circunstancias» ser también reivindicado, ejercido o disfrutado por grupos o por comunidades. ${ }^{15}$ Similar entendimiento ha tenido el Comité de Derechos Económicos, Sociales y Culturales del derecho a participar en la vida cultural. No obstante, esta interpretación no es extensible sin más a todos los derechos humanos. La dimensión colectiva de los derechos, es decir, que puedan ser practicados en grupo o comunidad, merece ser analizada caso a caso.

\footnotetext{
14. "Primer informe periódico de la experta independiente en la esfera de los derechos culturales», Naciones Unidas, A/HRC/14/36, 22 de marzo de 2010, párrafo 10, disponible en http://bit.ly/2RgTPqj.

15. «Observación General 17», Naciones Unidas, E/C.12/GC/17, 12 de enero de 2006, párrafo 8, disponible en http://bit.ly/2Ri5u8m.
} 
Un asunto diferente lo representan los derechos colectivos. Ellos constituirían una categoría especial -cuando menos excepcional- de derechos reconocidos en los instrumentos del derecho internacional. Como ejemplo paradigmático de derecho colectivo se cita el derecho a la libre determinación de los pueblos, recogido en el artículo 1 del Pacto Internacional de Derechos Civiles y Políticos. Esta norma no deja de ser controvertida, del mismo modo que los derechos colectivos de los pueblos indígenas, recogidos en la Declaración de las Naciones Unidas sobre los Derechos de los Pueblos Indígenas de 2007. En efecto, los derechos colectivos han sido generalmente resistidos por los Estados. Sus reservas se han sustentado en los «temores y sospechas» hacia su establecimiento, pues erróneamente se les asocia al secesionismo, sobre todo si se refieren a minorías o a pueblos indígenas (Symonides, 1998: 560). Como motivo de rechazo se han esgrimido también las tensiones políticas e ideológicas que pueden aflorar en casos en que los derechos de una persona entren en conflicto con los de un grupo (Nieć, 2001: 282-283).

Por mucho tiempo se estimó que la situación de las personas pertenecientes a minorías culturales, comprendiendo dentro de estas especialmente a los pueblos indígenas, concernía fundamentalmente a cada Estado y que siempre que existiera un respeto general hacia sus derechos humanos individuales, Naciones Unidas no debía asumir un rol de mayor responsabilidad. Además, la norma de la Carta Internacional de los Derechos Humanos más explícita en relación con las minorías, a saber, el artículo 27 del Pacto Internacional de Derechos Civiles y Políticos, se encuentra redactada de manera que no favorece una interpretación colectivista de sus derechos, por cuanto dispone que «en los Estados en que existan minorías étnicas, religiosas o lingüísticas», no se negará «a las personas que pertenezcan a estas minorías» el derecho a tener su propia vida cultural, a profesar y practicar su propia religión y a emplear su propio idioma, «en común con los demás miembros de su grupo».

Esta visión comenzó a cambiar hacia finales de los setenta, época en que representantes de algunos pueblos indígenas, ciertos delegados gubernamentales y miembros de la sociedad civil empezaron a llamar la atención en los foros internacionales sobre los continuados problemas de derechos humanos a los que se enfrentan las poblaciones indígenas alrededor del mundo. Como respuesta a estas inquietudes, en $1982 \mathrm{el}$ Consejo Económico y Social creó un grupo de trabajo sobre poblaciones indígenas, instancia que sentó las bases para que, 25 años después, la Asamblea General aprobara la Declaración sobre los Derechos de los Pueblos Indígenas. En el intertanto, la Organización Internacional del Trabajo adoptó el Convenio 169 sobre Pueblos Indígenas y Tribales de 1989, que sujeta a los Estados parte a efectuar una consulta previa, libre e informada en caso de medidas legislativas o administrativas que puedan afectar directamente a los pueblos indígenas. Este instrumento ha sido ratificado por poco más de veinte Estados, fundamentalmente de América Latina, pero no es claro que su aplicación sea efectiva. 
Ahora bien, la adopción de la Declaración de 2007 viene a asumir la situación de discriminación que los pueblos indígenas generalmente han sufrido en desmedro de sus derechos, por lo que apela a los Estados y a la comunidad internacional a dedicarles atención especial. Reafirma que las personas pertenecientes a pueblos indígenas gozan en lo individual de todos los derechos ya reconocidos a nivel internacional, pero además atribuye derechos colectivos debido a sus particularidades históricas y culturales. Estos derechos colectivos - establecidos de manera dispersa a lo largo de los 46 artículos que componen la Declaración- pueden ser agrupados en tres clases: los referidos a la libre determinación o a un estatus político de una autonomía que permita a estos pueblos afirmar y preservar su identidad; los asociados a sus tierras y recursos naturales; y los derechos de conservación y desarrollo de su cultura. ${ }^{16}$ Estos últimos bien podrían ser calificados de derechos culturales colectivos de los pueblos indígenas.

Este instrumento, que ya cumplió una década, ha contribuido a fortalecer la irrupción de las comunidades indígenas como actores sociales, políticos y culturales cada vez más relevantes. Busca proporcionar más herramientas jurídicas para el reconocimiento cabal de la condición jurídica propia del indígena, como individuo y especialmente como colectivo. Sin embargo, no se trata de una declaración vinculante para los Estados y puede ser vista como no más que soft law, aunque tiene el potencial de convertirse en derecho internacional consuetudinario con el tiempo si las prácticas nacionales, especialmente la jurisprudencia, la aplican consistentemente.

En definitiva, se puede apreciar que los derechos colectivos han sido resistidos políticamente, entre otros motivos porque pugnarían con los derechos individuales. A la vez, resultan difíciles de articular en forma teórica, por cuanto suponen definir precisamente al sujeto colectivo titular. A los pueblos indígenas se les han atribuido derechos colectivos debido a las particularidades históricas y culturales reconocidas por la comunidad internacional organizada.

Este tema excede ciertamente los límites del presente artículo. Para cerrar este acápite, se puede señalar que la dimensión colectiva del ejercicio de los derechos humanos y la cuestión de los derechos colectivos de los pueblos indígenas merecen ser vistas como mecanismos complementarios a los derechos expresamente culturales. Esto permite reafirmar el carácter eminentemente colectivo de la cultura, lo que enriquece y a su vez complejiza la perspectiva jurídica con que se tratan los asuntos culturales.

16. Para un panorama detallado de los derechos de los pueblos indígenas recogidos en la Declaración y en otros instrumentos del derecho internacional, véase Zalaquett (2008). 


\section{Derecho a participar en la vida cultural: Análisis general}

El derecho de toda persona a participar en la vida cultural de la comunidad ha sido visto como el derecho más obvio de los culturales. ${ }^{17}$ En la Observación General 21 de 2009, el Comité de Derechos Económicos, Sociales y Culturales ofrece una interpretación del contenido normativo del artículo 15, párrafo 1 letra a) del Pacto, disposición que contiene este derecho en dicho instrumento que obliga a sus Estados parte.

En primer lugar, el Comité interpreta que el derecho de toda persona a participar en la vida cultural es fundamentalmente una libertad. ${ }^{18}$ Para que la libertad cultural sea factible se necesita de parte del Estado que éste no interfiera en el ejercicio de las identidades culturales ni obstaculice el acceso a los bienes culturales y que, al mismo tiempo, proteja las prácticas o expresiones en la esfera de la cultura y mejore las condiciones materiales para una participación efectiva de todas las personas. El desempeño estatal respecto de este derecho tiene al menos dos dimensiones, abstención y actuación, que deben operar conjuntamente para su adecuada observancia. Esto ratifica la miopía de un enfoque sobre derechos humanos que establece una distinción tajante entre los derechos civiles y políticos, y los derechos económicos, sociales y culturales. Todos los derechos humanos universalmente reconocidos requieren de acciones positivas y negativas por parte del Estado, en virtud del «triple esquema de obligaciones» que conllevan: respetar, proteger y cumplir. ${ }^{19}$

Ahora bien, a la luz de una interpretación gramatical del artículo 15, párrafo 1, letra a) del Pacto, encontramos en primer lugar el fragmento «toda persona» que, para este caso, abarca tanto al sujeto individual como colectivo. En efecto, el Comité estima que con arreglo a quién lo ejerza, el derecho a participar en la vida cultural puede adoptar tres modalidades: ser ejercido por una persona, en forma individual; por una persona, en asociación con otras; o puede ser ejercido dentro de una comunidad o grupo.$^{20}$ Esto reafirma la dimensión colectiva del ejercicio del derecho en cuestión.

Luego está el verbo «participar»o «tomar parte», voces que han de ser interpre-

17. Así ha sido calificado por la relatora especial en su primer informe general de 2010 (párrafo 12). La doctrina también entiende este derecho como el más «amplio» del conjunto de derechos humanos culturales (Stamatopoulou-Robbins, 2008: 3-4).

18. «Observación General 17», párrafo 6.

19. La versión inicial del triple esquema de obligaciones es de Shue (1980), quien sostuvo que todo derecho básico exige del Estado: i) obligaciones de evitar privar el derecho; ii) obligaciones de proteger de la privación; y iii) obligaciones de auxiliar al privado de su derecho. Esta propuesta sería relevada entre otros por Eide (1989), quien las reformula en los siguientes términos: i) obligación primaria de respetar; ii) obligación secundaria de proteger; iii) obligación terciaria de cumplir. Para la evolución de esta tesis y su relación con la justiciabilidad de los derechos económicos, sociales y culturales, particularmente el derecho a la salud, véase Figueroa García-Huidobro (2009).

20. «Observación General 21», Naciones Unidas, E/C.12/GC/21, 21 de diciembre de 2009, párrafo 9, disponible en http://bit.ly/2ReHlzx. 
tadas indistintamente. En términos generales, la participación supone la libertad a elegir su identidad cultural, ejercer prácticas, tradiciones y formas de vida. Comprende el derecho de toda persona a buscar, desarrollar y compartir con otros sus conocimientos y expresiones culturales. ${ }^{21} \mathrm{El}$ verbo "participar» evoca entonces tanto el acceso como la contribución a la vida cultural.

La dimensión de acceso supone la posibilidad de cada persona de conocer y comprender su propia cultura, así como también la de otros. Ello es viable fundamentalmente a través de la educación y la difusión de la información (lo que conlleva el ejercicio de otros derechos humanos interrelacionados). Acceder a la cultura implica, además, disfrutar del patrimonio cultural, que según el Comité se desagrega en «conocer, comprender, entrar, visitar, utilizar, mantener, intercambiar y desarrollar el patrimonio cultural». Lo anterior lleva a comprender el acceso bajo la perspectiva del patrimonio cultural, en lugar del mero acceso a «productos» de la cultura. Los derechos culturales están más cerca de la idea de desarrollo cultural que de la noción de comercio o consumo. El aspecto de contribución, en tanto, alude a la facultad de toda persona de aportar en la creación y desarrollo de manifestaciones culturales de la comunidad, sean éstas expresiones artísticas, intelectuales, espirituales o de otra índole, así como en la formulación de políticas que incidan en el ejercicio de los derechos culturales.

El último elemento gramatical es «vida cultural». En palabras del Comité, esta expresión alude a la cultura como un «proceso vital, histórico, dinámico y evolutivo». Los sujetos culturales, personas y grupos, constituyen la expresión misma de la humanidad que se desenvuelve dentro de este proceso interactivo. Se trata entonces de un concepto que remite fuertemente a lo colectivo, si tenemos en cuenta la individualidad y la alteridad que supone la cultura como creación y producto social.

En síntesis, este derecho consiste en una libertad reconocida a todo ser humano, que puede ser ejercida individualmente, con otras personas o dentro de una comunidad o grupo. Además, esta libertad supone tanto acceso como contribución a la vida cultural, por lo que el derecho en cuestión cubre tanto una participación pasiva («beneficiarse» o «gozar») como una más activa («contribuir» o «crear»). Esta interpretación asume adecuadamente que las personas son a la vez espectadores y creadores de la cultura.

21. Esto es concordante con la definición de participación en la vida cultural propuesta en la Recomendación relativa a la Participación y la Contribución de las Masas Populares en la Vida Cultural de la Unesco de 1976, en el que se entiende como «la posibilidad efectiva y garantizada para todo grupo o individuo de expresarse, comunicar, actuar y crear libremente, con objeto de asegurar su propio desarrollo, una vida armoniosa y el progreso cultural de la sociedad». 


\section{Obligaciones de los Estados para respetar, proteger y cumplir este derecho}

Los derechos humanos se ejercen erga omnes, por lo que generan obligaciones para y respecto de todas las personas. Sin embargo, desde la perspectiva del derecho internacional de los derechos humanos, son los Estados los principales obligados y garantes. El Pacto Internacional de Derechos Económicos, Sociales y Culturales expresa que las obligaciones estatales consisten en adoptar medidas, hasta el máximo de los recursos disponibles, que tiendan a lograr progresivamente su plena efectividad (artículo 2, inciso primero). Como se ha advertido, se suele hacer énfasis en el aspecto progresivo de estos derechos, pero una adecuada interpretación general ha de asumir que todo derecho reconocido esencial al ser humano involucra un conjunto de obligaciones de los Estados para observarlos y asegurar un nivel de satisfacción mínimo. ${ }^{22}$

El derecho a participar libremente en la vida cultural exige del Estado mucho más que solo deferencia hacia su ejercicio: supone un despliegue sustantivo que haga posible su más plena realización. El contenido de la actuación del Estado varía en función de las distintas clases de obligaciones que se identifican. El Comité sigue el triple esquema de obligaciones de respeto, protección y cumplimiento, por lo cual traza acciones o medidas concretas a efectuar por el Estado para cada caso, así como comprende que este derecho requiere de un nivel mínimo de satisfacción, sin el cual no tendría contenido alguno y se convertiría en un mero enunciado teórico. ${ }^{23}$

En primer lugar, la obligación de respeto del Estado hacia el derecho de participar en la vida cultural requiere que se abstenga de interferir, directa o indirectamente, en la esfera de libertad de participación en la vida cultural, libertad de las personas de escoger y desarrollar sus identidades culturales, individualmente y en grupo, lo que a su vez supone la facultad de proporcionar, reunir y transmitir información e ideas en la esfera de la cultura. Así, la obligación de respetar es en buena medida una obligación negativa, que exige observar la libertad de participación, lo que conlleva el respeto por otras libertades especialmente interrelacionadas, a saber, las de opinión, expresión, información y creación.

La obligación general de protección, por su parte, requiere que los Estados parte adopten medidas positivas para impedir que otros agentes interfieran en el ejercicio de este derecho. Es decir, significa un resguardo respecto de las acciones de terceros que puedan perturbar el disfrute del derecho. Esto comprende las injerencias tanto de

22. El Comité ha venido desarrollando sostenidamente esta interpretación desde su Observación General 3 de 1990, referida precisamente a la índole de las obligaciones de los Estados respecto de los derechos económicos, sociales y culturales.

23. Para el análisis detallado del Comité respecto de las diversas clases de obligaciones que conciernen a los Estados parte del Pacto Internacional de Derechos Económicos, Sociales y Culturales en virtud del derecho dispuesto en el artículo 15, párrafo 1, letra a), véase la «Observación General 21», párrafos 44-59. 
organismos públicos como privados, así como también las externas: por ejemplo, influencias extranjeras nocivas que propendan hacia la uniformización de la cultura en desmedro de las identidades culturales locales. El Estado debe proteger, además, los intereses morales y materiales que se derivan de la actividad creativa, lo cual supone la protección de estos derechos ante las eventuales vulneraciones por parte de otros individuos. La obligación de proteger también comporta la preservación y salvaguarda del patrimonio cultural para las generaciones venideras. En efecto, el Comité precisa que las obligaciones de respetar y proteger las libertades, el patrimonio cultural y la diversidad están fuertemente interrelacionadas y ahonda en medidas vinculadas a esta obligación.

Por último, la obligación de cumplimiento o satisfacción exige del Estado la adopción de medidas activas (legislativas, administrativas, judiciales, presupuestarias y de promoción) que provean las condiciones para una amplia participación en la vida cultural por parte de todas las personas. La orientación de las medidas tendientes a satisfacer el derecho puede comprenderse mejor a la luz de la subdivisión de la obligación de satisfacer en las de facilitar, promover y proporcionar.

La obligación de facilitar consiste en fortalecer la capacidad de todas las personas para ejercer y satisfacer su derecho a participar en la vida cultural, mientras que la de promover radica en medidas de divulgación y educación sobre el derecho a la cultura y sus implicaciones. En este comportamiento activo, el Estado debiese atender especialmente a la población más vulnerable, que incluye personas en situación de pobreza, minorías y pueblos indígenas. La promoción necesita también de una educación enfocada en el valor de la diversidad humana y el patrimonio cultural. Por último, la obligación de proporcionar consiste en la adopción de medidas positivas que hagan posibles las condiciones materiales para que toda persona pueda participar efectivamente en la vida cultural. Esto requiere de programas que provean de acceso no discriminatorio a la más amplia gama de bienes y servicios culturales que sea posible, especialmente a través de sus medios institucionales, como museos o bibliotecas.

En cuanto al contenido mínimo del derecho a participar en la vida cultural, el Comité ha expresado que consiste en «crear y promover un entorno en el que toda persona, individualmente, en asociación con otros o dentro de una comunidad o grupo, pueda participar en la cultura de su elección». ${ }^{24}$ El nivel mínimo de satisfacción comprende cinco obligaciones básicas (core obligations): i) garantizar la no discriminación; ii) respetar la libre elección de identidades culturales; iii) respetar y proteger derecho a ejercer las prácticas culturales legítimas; iv) eliminar las barreras que impidan o limiten el acceso de la persona a su propia cultura o a otras culturas; v) permitir y promover la participación de personas pertenecientes a minorías, pue-

24. «Observación General 21», párrafo 55. 
blos indígenas y otras comunidades en la formulación y aplicación de las leyes y las políticas que les atañan.

Ahora bien, las violaciones al derecho a participar en la vida cultural pueden ocurrir por la acción u omisión tanto del Estado como de terceros. Las maneras de vulnerar este derecho son múltiples, pero un modo general y sistemático sobreviene precisamente si los Estados no logran adoptar las medidas elementales que permitan asegurar un nivel básico de satisfacción. También existe violación grave cuando una acción menoscaba deliberadamente el ejercicio de alguno de los elementos fundamentales del derecho. En este sentido, los actos iconoclastas y biblioclastas constituyen maneras evidentes de destruir intencionalmente el patrimonio cultural. Este tipo de acciones buscan arruinar bienes que producen identificación cultural y en definitiva lesionan el derecho a la cultura.

Los Estados tienen el deber de proveer mecanismos judiciales y administrativos eficaces que hagan justiciables estos derechos ante vulneraciones. Sin embargo, se constata una falta de medios idóneos para la exigibilidad de los derechos económicos, sociales y culturales en general. Pero de la ausencia de herramientas adecuadas para remediar la violación de obligaciones que tienen como fuente estos derechos, no se sigue de ningún modo la imposibilidad técnica de crearlas y desarrollarlas (Abramovich y Courtis, 2002: 40). Y en casos calificados de violaciones o ante una nula protección interna, ha de contemplarse la posibilidad de acudir a instancias internacionales. Aquí cabe hacer referencia al Protocolo Facultativo del Pacto Internacional de Derechos Económicos, Sociales y Culturales, instrumento que establece un mecanismo de denuncias en caso de violaciones a estos derechos humanos. En este procedimiento, el Comité de Derechos Económicos, Sociales y Culturales media entre la persona y el Estado parte respectivo con el objeto de arribar a una solución. ${ }^{25}$

Lamentablemente, este instrumento ha sido ratificado solo por una veintena de países, hecho relacionado con las obligaciones internacionales de los Estados, sujetos del derecho internacional que se comprometen a cumplir los tratados que suscriben. El Pacto Internacional de Derechos Económicos, Sociales y Culturales estipula también que los Estados parte convienen en adoptar medidas de orden internacional

25. Al Comité se le faculta para estudiar cada denuncia o «comunicación» de toda persona o grupo de personas que alegue ser víctimas de una violación de un Estado parte de cualquiera de los derechos económicos, sociales y culturales establecidos en el pacto respectivo. Dicha comunicación debe superar un examen de admisibilidad que exige el «agotamiento de los recursos internos». Declarado admisible, el Comité podrá eventualmente solicitar al Estado parte la adopción de medidas provisionales y pondrá sus buenos oficios a disposición de las partes con el objeto de llegar a una solución amigable. En caso de no tener éxito, el Comité examinará el fondo de la denuncia, junto con la documentación ofrecida por ambas partes, para luego pronunciar un dictamen, que podrá contener recomendaciones. En este caso, el Estado parte deberá responder en un plazo de seis meses proveyendo información sobre toda medida que haya adoptado a la luz del dictamen y las recomendaciones efectuadas. 
tendientes a asegurar el respeto de los derechos que en él se reconocen, lo que comprende la implementación de las recomendaciones de los organismos pertinentes de Naciones Unidas. En virtud de lo anterior, ha de entenderse que la esfera de la cultura debe ser realmente incorporada en el ámbito de acción de las relaciones internacionales. Además, las organizaciones internacionales tienen la responsabilidad de «asegurar que los derechos culturales y la dimensión cultural de los derechos humanos sean tomados en consideración de manera sistemática», como se afirma en el artículo 12 de la Declaración de Friburgo. Sin dudas queda mucho por hacer en este sentido, pues los derechos culturales siguen reclamando mayor visibilidad e incidencia en los instrumentos internacionales de desarrollo y cooperación.

\section{Conclusiones}

Se ha intentado ofrecer una aproximación general a los derechos culturales sobre la base de una interpretación de las normas de la Carta Internacional de Derechos Humanos referidas explícitamente a la esfera de la cultura. Se ha insinuado el amplio espectro de cuestiones del ámbito de la cultura que tienen repercusiones jurídicas y se ha planteado que éstas puedan ser consideradas desde el catálogo acotado de derechos expresamente culturales que se propone. De entre aquellas, se han abordado las nociones de identidad, patrimonio y diversidad cultural, que resultan especialmente relevantes en el discurso alrededor de estos derechos, así como para las obligaciones que conllevan. Adicionalmente, se ha aludido al ejercicio en grupo o comunidad de los derechos individuales y a la existencia de los derechos colectivos, asuntos complejos que merecen ser vistos como complementarios al ejercicio de los derechos humanos individuales en la esfera de la cultura. Posteriormente, se analizó el derecho de toda persona a participar en la vida cultural, cuyo contenido y alcance se estima como el más amplio y representativo de esta categoría de derechos humanos. Finalmente, se ha hecho énfasis en las obligaciones que se desprenden para el Estado con relación al respeto, protección y cumplimiento de este derecho cultural.

Se constata que si bien los derechos culturales han sido universalmente reconocidos en los instrumentos más relevantes del derecho internacional de los derechos humanos, solo en tiempo reciente han comenzado a ser estudiados con mayor interés. En los últimos años se ha avanzado bastante en términos de su clarificación normativa y cada vez hay más conciencia del real peso que tienen. Los derechos culturales - para parafrasear a un autor que retrata así al derecho a la ciencia - son «viejos derechos, pero con mucho futuro» (Mancisidor, 2017). Mientras se hace evidente que se trata de derechos indispensables para el respeto de la dignidad de todas las personas y grupos, de a poco se va asumiendo el gran potencial que tienen en la resolución de los conflictos humanos y el rol de paz que cumplen. En efecto, estos derechos «protegen el desarrollo y la expresión de diversas visiones del mundo —individuales 
y colectivas- y abarcan importantes libertades relacionadas con cuestiones de identidad», como bien los caracteriza la relatora especial, quien añade que ellos constituyen «instrumentos esenciales del desarrollo, la paz y la erradicación de la pobreza, la consolidación de la cohesión social y el respeto de la comprensión recíproca entre individuos y grupos, en toda su diversidad». ${ }^{26}$

Esta categoría de derechos humanos ha ido paulatinamente superando los obstáculos conceptuales que les impedían gozar de la misma posición teórica y jurídica que otros derechos humanos. Sin embargo, no es posible afirmar lo mismo respecto de su implementación efectiva, donde todavía resta bastante por hacer. De hecho, aún continúan siendo relativamente ignorados por los Estados. En general, se advierte una escasa inclusión del ámbito de la cultura a nivel constitucional, ya que es todavía más difícil encontrar derechos culturales - al margen del derecho a la educación- establecidos en términos de derechos fundamentales.

Tampoco la comunidad internacional organizada los ha incorporado adecuadamente en sus ámbitos de acción más relevantes. Esto se confirma si se considera el instrumento reciente más significativo de Naciones Unidas sobre el desarrollo, la Agenda 2030 para el Desarrollo Sostenible. Este instrumento contempla nulas referencias a los derechos culturales, únicamente el párrafo 36 de la resolución que aprueba la Agenda se refiere al «entendimiento de las culturas» y la «diversidad cultural». Ninguno de los diecisiete objetivos de desarrollo sostenible propuestos alude a la esfera de la cultura o a la vida cultural. Esta omisión parece responder a distintos motivos, entre ellos la falta de voluntad que todavía se aprecia por parte de los Estados para aceptar, más allá de una cierta corrección política, las implicaciones de la diversidad cultural. Adicionalmente, se evidencia que, por alguna razón política al interior de la estructura de las Naciones Unidas, la Unesco se ha visto impedida de ejercer un liderazgo fuerte en esta materia, del mismo modo que su labor no ha sido suficientemente tomada en cuenta por los Estados.

Lo anterior demuestra que los derechos culturales siguen siendo una categoría subestimada de derechos humanos. Es por ello que se hace necesario perseverar en su promoción y realización, lo que supone también seguir investigándolos. Estos esfuerzos son urgentes, por cuanto los derechos culturales juegan un rol fundamental en el desarrollo integral de las personas y las sociedades. Ellos permiten resguardar los diversos modos de vida y visiones de mundo, tanto a partir de las identidades individuales como colectivas. La vida cultural constituye el espacio en que las diversas identidades humanas se desenvuelven y se relacionan, lo que da lugar a las expresiones simbólicas y materiales que producen sentido tanto a quienes las practican como a quienes las observan. Hay entonces que reivindicar el rol de los derechos culturales, especialmente del derecho a participar en la vida cultural. El establecimiento de un

26. «Primer informe periódico», párrafo 3 . 
adecuado marco para la libertad de participación dentro de la esfera de la cultura fija las condiciones para el debido respeto por la diversidad humana y la preservación de los bienes patrimoniales, mientras que el fomento a la creatividad y a las manifestaciones de la cultura - entre ellas las producciones científicas, artísticas y literariaspermite reforzar las potencialidades de la realidad social, con el fin de empoderar a las personas en los procesos de desarrollo humano.

\section{Agradecimientos}

El autor agradece a Francisco Acosta Joerges por los valiosos comentarios hechos a una versión preliminar de este artículo.

\section{Referencias}

Abramovich, Víctor y Christian Courtis (2002). Los derechos sociales como derechos exigibles. Madrid: Trotta.

DonDers, Yvonne (2004). «El marco legal del derecho a participar en la vida cultural». En Agencia Española de Cooperación Internacional para el Desarrollo, Derechos culturales y desarrollo humano (pp. 153-170). Madrid: AECID.

-. (2008). "Cultural life in context of human rights». Documento de trabajo presentado al Comité de Derechos Económicos, Sociales y Culturales de las Naciones Unidas para su cuadragésima sesión, Ginebra. E/C.12/40/13. Disponible en http:// bit.ly/2CKaSob.

EIDE, Asbjørn (1989). «Realization of social and economic rights: the minimum threshold approach». International Commission of Jurists Review, 43: 40-52.

Figueroa García-Huidobro, Rodolfo (2009). «Justificación del rol de las cortes haciendo justiciables los derechos económicos, sociales y culturales, en particular, el derecho a la protección de la salud». Revista Chilena de Derecho, 36 (2):313-342. DOI: $10.4067 /$ So718-34372009000200005.

MANCisidor, Mikel (2017). «El derecho humano a la ciencia: Un viejo derecho con un gran futuro». Anuario de Derechos Humanos, 13: 211-221. DOI: 10.5354/0718-2279.2017.46887.

Meyer-Bisch, Patrice (2010). «Définir les droits culturels». Documento de trabajo para Seminario sobre la Implementación de los Derechos Culturales, organizado por la Oficina del Alto Comisionado de Derechos Humanos, Ginebra. Disponible en http://bit.ly/2CH8ya2.

MuÑoz CÁrCAmo, Andrés (2016). «Derechos culturales en cuanto derechos humanos: Aproximaciones a su contenido y alcance desde el derecho internacional». Memoria de grado para optar a la Licenciatura en Ciencias Jurídicas y Sociales. Universidad de Chile. Disponible en http://bit.ly/2KWCSzz. 
Nieć, Halina (2001). «Sentar las bases para la realización de los derechos culturales». En Unesco, ¿A favor o en contra de los derechos culturales? París.

Prieto de Pedro, Jesús José (2008). «Derechos culturales, el hijo pródigo de los derechos humanos». Crítica, 58 (952): 19-24. Disponible en http://bit.ly/2SmLxio.

Shue, Henry (1980). Basic rights, subsistence, affluence and U.S. foreign policy. Princeton: Princeton University Press.

Stamatopoulou-Robbins, Elissavet (2008). «The right to take part in cultural life». Documento de trabajo presentado al Comité de Derechos Económicos, Sociales y Culturales de las Naciones Unidas para su cuadragésima sesión, Ginebra. E/C.12/40/9. Disponible en http://bit.ly/2CHFYVT.

Symonides, Janusz (1993). «The history of the paradox of cultural rights and the state of the discussion within Unesco». En Patrice Meyer-Bisch (editor), Les droits culturels: Une catégorie sous-développée de droits de l'homme. Friburgo: Éditions Universitaires Fribourg Suisse.

-. (1998). «Cultural rights: A neglected category of human rights». International Social Science Journal, 50 (158): 559-572. DOI: 10.1111/1468-2451.00168.

Teixeira Coelho, José (2008). «Brindando concreción a los derechos culturales». En Daniela Bobbio (compiladora), Tensiones: Selección de Conferencias del Programa de Formación en Gestión Cultural. Córdoba: Centro Cultural de España.

VAšÁk, Karel (1977). «La larga lucha por los derechos humanos». El Correo de la Unesco, 30 (11): 29-32. Disponible en http://bit.ly/2CHCIdd.

Zalaquett, José (2008). «La Declaración de Naciones Unidas sobre Derechos de los Pueblos Indígenas». Anuario de Derechos Humanos, 4: 139-148. DOI: 10.5354/0718-2279.2011.13508.

\section{Sobre el autor}

Andrés Muñoz CárCamo es abogado. Licenciado en Ciencias Jurídicas y Sociales de la Universidad de Chile. Cursó el Diplomado en Relaciones Culturales Internacionales impartido virtualmente por la Organización de Estados Iberoamericanos para la Educación, la Ciencia y la Cultura a través de la Universidad de Gerona. En 2017 siguió una pasantía en el Alto Comisionado de Naciones Unidas para los Derechos Humanos, en su Oficina Regional para América del Sur. Su correo electrónico es andresmunozcar@gmail.com. 
El Anuario de Derechos Humanos es una publicación anual de referencia y consulta en derechos humanos y materias afines, que busca ser un espacio de discusión, difusión y conocimiento de los temas centrales sobre derechos humanos en sus contextos nacional e internacional, poniendo a la disposición del público de manera gratuita los distintos desarrollos doctrinales, jurisprudenciales y legislativos ocurridos en este campo dentro del período anual cubierto por cada edición.

\author{
DIRECTORA RESPONSABLE \\ Nancy Yáñez Fuenzalida \\ EDITOR DE CONTENIDOS \\ Salvador Millaleo \\ SITIO WEB \\ anuariocdh.uchile.cl \\ CORREO ELECTRÓNICO \\ anuario-cdh@derecho.uchile.cl
}

LICENCIA DE ESTE ARTÍ́CULO

Creative Commons Atribución Compartir Igual 4.0 Internacional

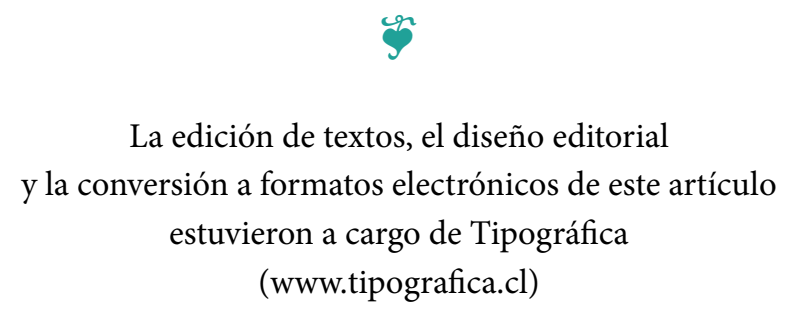

